\title{
Influence of Clothing Materials on Protective Performance in Tennis
}

\author{
Yuan $\mathrm{Li}^{1}$, Hong Xie ${ }^{1} \&$ Hongqiong Deng ${ }^{1}$ \\ ${ }^{1}$ Fashion college, Shanghai University of Engineering Science, Shanghai, China \\ Correspondence: Yuan Li, Address: School of Fashion Technology, Shanghai University of Engineering Science, \\ Shanghai, 201600, China
}

Received: July 6, 2017

Accepted: July 27, 2017

Online Published: August 18, 2017

doi:10.5430/jbar.v6n2p27

URL: https://doi.org/10.5430/jbar.v6n2p27

\begin{abstract}
The main objective of this study is to analyze influence of clothing materials on protective performance in tennis sportswear. Firstly, the paper presented a human protection model on tennis, and deduced two evaluation parameters to describe protection performance. Secondly, the experimental objects wore different gears made by different fabrics for tennis serve and the experiment data was collected to analyze the gears' protection performance. Thickness, elasticity, fabric composite methods and wrapping types were set as independent variables of the gears. The ANOVA shows that these factors are of great significance to change the evaluation parameters of the upper limbs joints. Thickness of fabrics is more significant $(\mathrm{P}<0.01)$ on evaluation parameters than that of elasticity, especially for elbow $(\mathrm{P}<0.01)$, while elastic of fabric only affects peak momentum of elbow and wrist, but not obvious. The wrapping ways of pads are important factors to peak momentum changes. This research shows clothing material is an important element to design protective tennis gears.
\end{abstract}

Keywords: Clothing materials, Protective performance, Upper limb joints, Tennis

\section{Introduction}

A recent study of professional tennis competitions found that more than $50 \%$ of men's and women's departures from competition could be attributed to injury (Okholm et al 2015). Sports injuries in tennis athletes are common, expercially for competitive tennis, it involves extensive training and frequently repeated motions, on which all tennis strokes apply high musculoskeletal loads (Elliott et al, 2003; Escamilla \& Andrews, 2009). Such loads elicit sports-specific adaptive responses of dominant upper limb bones and muscles (Sanchis et al, 2012). Meanwhile, the continuous use of the upper extremities in producing force and direction to the ground strokes leads to repetitive overuse and high levels of mechanical and physiological stress on muscles, tendons and joints that often result in injury (Ellenbecker, 1991).

People in motion are to consciously wear sports protective gears to avoid damage. The British scientist Richard Palmer developed d3o (Palmer \& Green, 2010) that can protect athletes from impact injury. it is used to make products such as ice skating pants and suits for athletes. Dow Corning developed an active protection system, which used a 3D fabric liner treated with silicone rubber, it gets hard when suffer impact and return to soft after impact dissipation (Graham, 2006). The principle of impact resistant protective products is that apply the materials of damping capacity into the clothing or textiles, which are used to absorb the sudden impact of collision to protect the human body (Wang et al, 2013).

However, there is no complete evaluation standards that explain how protective products function. Developing protective products is a multidisciplinary process, which involve clothing materials mechanics, ergonomics and other discipline. This paper focused on the influence of clothing materials and proposed the research methods involving biomechanical, dynamic measurement, material properties analysis to study clothing materials on designing protective sportswear.

\section{Methods}

\subsection{Sports injury Analysis in Tennis}

Tennis strokes mobilise a kinetic chain involving the transfer of the energy generated by legs and trunk to the dominant upper limb, and finally to the racket (Sciascia et al, 2012), shock was transmitted to the player's forearm (Wei et al, 2006) during this process. In a single tennis match, this cycle is repeated numerous times and relies heavily on an athlete's strength, endurance, flexibility, and technique(Johnson et al, 2006) (Kovacs, 2006). Tennis 
injuries have a complex association with biomechanical properties of tennis strokes and serves. Specific and dynamic upper extremity positioning can account for large amounts of the speed at impact and varies by stroke type (Chung \& Lark, 2017). When the ball collides with the racket, shock and vibration load were transferred to the arm which is the most vulnerable to human bones and joints. Joint damage is likely to happen when the external shock time is too short (0.001s), because synovial fluid isn't ready to react to buffer this load (Editing Group for Sports Biomechanics, 2000), which results in joint damage. According to statistics, the time of tennis strokes is only $0.004 \mathrm{~s}$, the human joints is relatively vulnerable to injury at this state.

\subsection{Sports Injury Mechanism}

Bones or joints have their inherent limit, once the external force caused by movement is over the human body's endurance, it will cause sports injuries (Velani et al, 2012). In motion, Man's bones are unable to accept direct impact, the external force is usually dissipated through the muscle tissue and external buffer, thus human body can obtain some protection. Therefore, mechanism of the body protection in motion mainly lies in: reducing the external force on the human body, caused by balls, equipment and others or enhancing muscle protection ability through continuous training that can protect bones and joints.

\subsection{Human Body Movement Protection Model}

Muscle has its certain limit, when the external load is over the critical value, the external impact will cause damage on the skeletal joints. whereas muscle damage may affect its function, easily resulting in secondary damage. To avoid such sports injuries, the body needs to wear external auxiliary products to dissipate external force. So this study combining the spring oscillator and the muscular mechanical performance to explain a basic model of human motion protection (Fig. 2) . The muscles and auxiliary product form a new protective layer to buffer the external load (muscles and external auxiliary are seen as an integration, shearing and other factors was not taken into consideration in this paper. ).

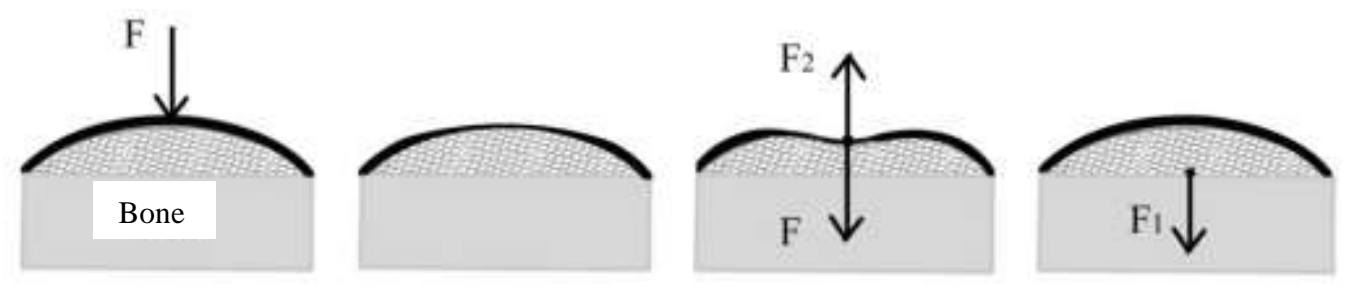

Figure 2. human protection model.

Rectangle represents human body parts, while semicircle represents the new protection layer of muscles and the fabric

$$
\begin{gathered}
F_{2}=K^{\prime} *\left(L-L_{1}\right) \\
F_{1}=F-F_{2}=F-K^{\prime} *\left(L-L_{1}\right)
\end{gathered}
$$

Where $F$ is the external force, and $F_{1}$ is the force acted on bones after muscle dissipation, $F_{2}$ is the buffer force. $K$ denotes compression modulus of muscle; $L$ is the original length, $L_{1}$ is the muscle length after compression (perpendicular to the surface of the muscle).

Assuming the quality of tennis is $m$, the ball's velocity is $v$ before impact and $v^{\prime}$ after impact, the force $F$ that the ball exerts to the racket is perpendicular to the racket. In order to simplify the model, $F$ is seen as a direct force on the body. Therefore, when the ball strikes on the racket, it gives the protective layer an initial velocity $v_{0} . F$ makes the protective layer deform, then the protection layer stores energy in this process. When the deformation reaches its maximum, the fabric layer begins to rebound, the protective layer can be converted to achieve the effect of the buffer, while the remaining force $F_{1}$ is transmitted to the human body.

$F_{2}$ relates to the elastic module of the protection layer, so the relationship between the layer and the biomechanical parameters can be established via $K^{\prime}$. Assuming velocity of the protection layer change from $v_{0}$ to $v^{\prime}$ during $t_{1}$. According to momentum theorem: 


$$
F_{2}=\frac{m v_{0}-m v^{\prime}}{t}
$$

$F_{2}$ reflects the protective performance of the protection layer. The larger the force $F_{2}$, the less the damage to the joints. In the line graph $m v-t$, the change rate of momentum is referred to momentum buffer coefficient (MBC) in this paper. The evaluation parameters of the fabric protection performance in this paper are determined as the momentum and the momentum buffer force after impact. Then Eq. (4) can be converted to:

$$
\begin{aligned}
& P=F t-F_{1} t \\
& P=m a t-K^{\prime} *\left(L-L_{1}\right) * t=m v-K^{\prime} *\left(L-L_{1}\right) * t
\end{aligned}
$$

In Eq. (5), $P$ is the momentum of each joint after impact; $m v$ is the tennis ball's momentum the moment of impact.

The fabric-covered muscle formed a new protection layer, The value $F_{2}$ was larger, the protective performance of the protective layer was better.

\section{Experiments and Results}

\subsection{Protective Performance Tests on Clothing Materials}

This paper selected NDI Optotrak system for three-dimensional dynamic action capture. Set tennis serve as experimental action. The measured person stood at a distance of 5 meters from the effective point, and the ball was recorded as a valid experiment within the effective range (Radius of $10 \mathrm{~cm}$ circle). Four rigid bodies was set, each rigid body consisted of three marker points. The rigid body positions were distributed in the middle of the arm, the middle of the forearm, the waist and the back neck. According to the guidance of the NDI company, five virtual points were set, namely the scapula protrusion point and the elbow inner and outer sides (Tab. 1).

Table 1. Rigid body and corresponding names of the virtual point

\begin{tabular}{lll}
\hline Rigid body & Corresponding part & Virtual point name \\
\hline \multirow{2}{*}{ Upper arm } & Shoulder (The lateral and interior), & SP ( Shoulder point), EI (The elbow interior), \\
& Elbow (The lateral and interior) & EL (The elbow lateral) \\
Forearm & Wrist (The lateral and interior) & WI (The wrist interior), WL (The wrist lateral) \\
\hline
\end{tabular}

\subsubsection{Protection Performance Test on Thickness and Elastic}

Clothing's protective performance is mainly through pressure generated by dressing to help share the joint ligament load. It also works through constriction, pressure or support to enhance the stability of joints, to help joints scattered too much improper force. The constriction and pressure are mainly reflected in the elasticity and thickness of the fabric. This experiment aimed at verifying the influence of clothing material for protecting the human joint.

Shoulder pads, elbow pads and wristbands (abbr. S, E, W) were made by different fabrics. Six experimental objects were wearing different gears for tennis serve. The protection performance of each gear would be evaluated by the two parameters (Momentum, MBC), and told if two factors (elastic and thickness) influence the performance of fabrics. Each gear would not exceed three fabric layers (1 layer, 2 layers, 3layers). The basic parameters of each fabric were measured before experiment (Tab. 2).

Table 2. Experiment materials basic parameters

\begin{tabular}{lllll}
\hline & & \multicolumn{2}{l}{ Elastic recovery $(\%)$} \\
\cline { 4 - 5 } Fabric & Component & Thickness $(\mathrm{mm})$ & Warp & Weft \\
\hline A & 20\% Spandex, 80\% Nylon & 0.6 & 69.56 & 94.82 \\
B & 16\% Spandex, 84\% Nylon & 0.6 & 75.16 & 90.17 \\
C & 16\% Spandex, 84\% Nylon & 0.6 & 81.61 & 89.26 \\
D & 19\% Spandex, 81\% Nylon & 0.6 & 90.75 & 94.61 \\
E & 15\% Spandex, 85\% Nylon & 0.6 & 95.17 & 96.37 \\
\hline
\end{tabular}

Experimental objects worn 45 (five fabrics, three styles, three layers) protective gears doing dynamic measurement experiment, collecting human upper limb joints three-dimensional spatial data. The objects had a break for $10 \mathrm{~min}$ after three experiment actions. The intuitive line charts are shown in Fig. 4, Fig. 5. 


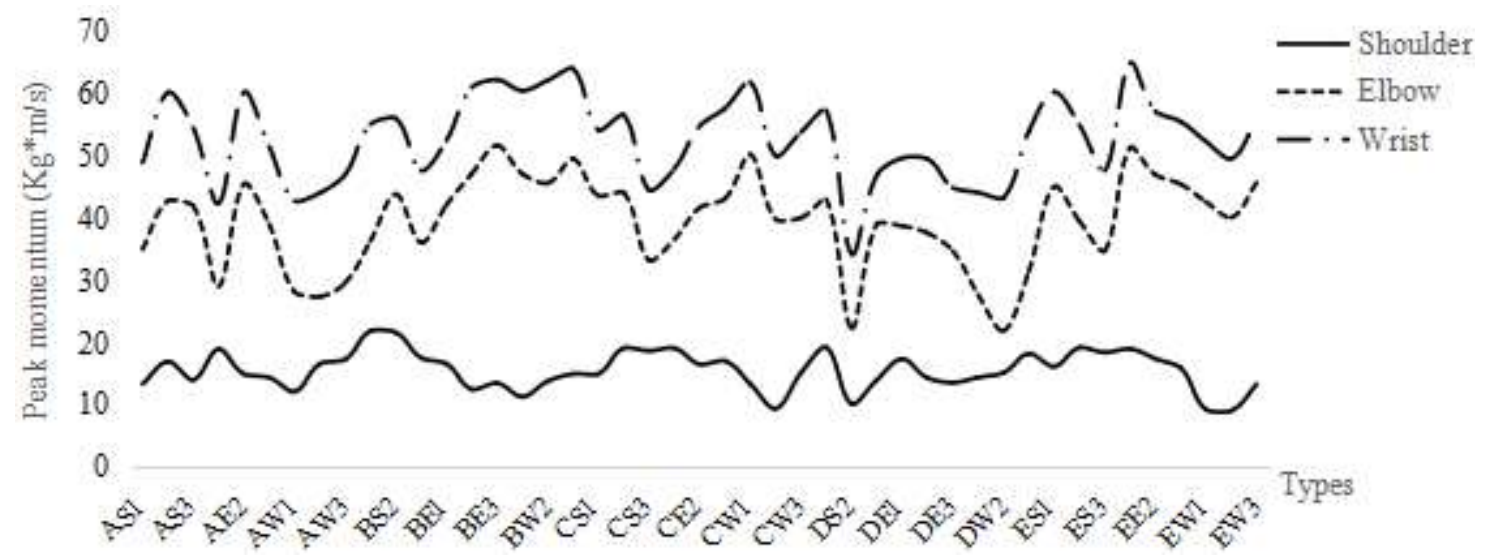

Figure 3. Peak momentum of upper joints changes with different protective pads

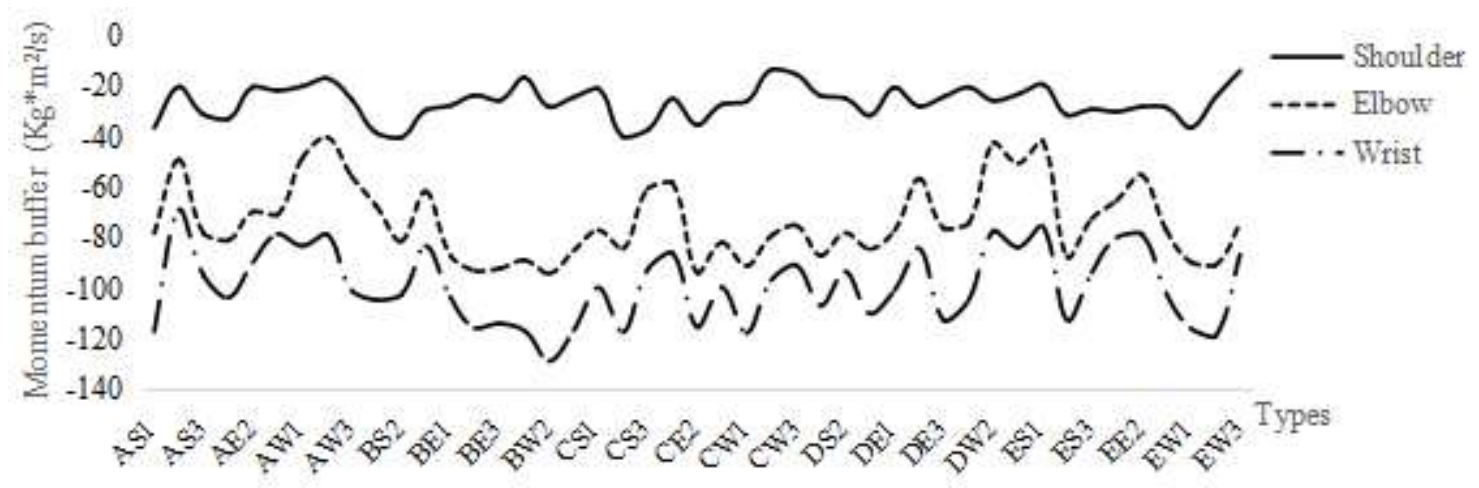

Figure 4. Momentum buffer coefficient of upper joints changes with different protective pads

To verify fabric elasticity's influence on the momentum and $\mathrm{MBC}$ in the upper limb, the amount of the corresponding change in the joints was compared with the same thickness.

When elastic changes, the peak momentum of the upper limb joints vary. The change trend in elbow and the wrist is consistent, while change line of shoulder fluctuates greatly that explains the function of shoulder pads. The MBC has changed especially in elbow and wrist after wearing gears. As for thickness, the peak momentum show negative correlation with it, the thicker the gears, the smaller the value of the peak momentum, but not for every fabric, which may relate to elasticity. The change of fabric thickness has some influence on MBC, especially A, C and E.

\subsubsection{Comprehensive Evaluation}

In order to tell which factor is of greater significance between elastic and thickness on momentum and MBC of the upper limb, this paper used the one-way ANOVA in SPSS to analyze whether the two factors had significance. Tab. 3 and Tab. 4 show the results of single factor analysis of variance (confidence interval 95\%). The elasticity factor and thickness factor are independent variables, the momentum and $\mathrm{MBC}$ of each joint in the upper limb are strain variables, Value $\mathrm{P}$ is significance. 
Table 3. Joint evaluation parameter changes with elasticity factor

\begin{tabular}{llll}
\hline Evaluation parameter & Value P ( 1 layer) & Value P ( 2 layer) & Value P ( 3 layer) \\
\hline Shoulder-M & 0.940 & 0.912 & 0.847 \\
Elbow-M & 0.056 & 0.042 & 0.302 \\
Wrist-M & 0.080 & 0.055 & 0.464 \\
Shoulder-MBC & 0.652 & 0.419 & 0.613 \\
Elbow-MBC & 0.744 & 0.040 & 0.843 \\
Wrist-MBC & 0.671 & 0.035 & 0.668 \\
\hline
\end{tabular}

Table 4. Evaluation parameter changes with thickness factor

\begin{tabular}{llllll}
\hline Evaluation parameter & Value P $(\mathrm{A})$ & Value $\mathrm{P}(\mathrm{B})$ & Value $\mathrm{P}(\mathrm{C})$ & Value $\mathrm{P}(\mathrm{D})$ & Value $\mathrm{P}(\mathrm{E})$ \\
\hline Shoulder-M & 0.003 & 0.039 & 0.012 & 0.063 & 0.032 \\
Elbow-M & 0.071 & 0.002 & 0.021 & 0.037 & 0.087 \\
Wrist-M & 0.049 & 0.047 & 0.037 & 0.176 & 0.001 \\
Shoulder-MBC & 0.004 & 0.087 & 0.024 & 0.007 & 0.040 \\
Elbow-MBC & 0.033 & 0.042 & 0.013 & 0.044 & 0.052 \\
Wrist-MBC & 0.038 & 0.157 & 0.069 & 0.150 & 0.020 \\
\hline
\end{tabular}

According to Tab. 3, when thickness changes, the evaluation parameters have significant changes, but some are not obvious, such as $\mathrm{MBC}$ of wristband with fabric $\mathrm{B}$, the momentum and $\mathrm{MBC}$ of wristband with fabric D. Its significant value is even greater than 0.1. In a word, the evaluation parameters of the wrist is not significant when thickness changes. From the analysis, thickness of fabric has a significant effect on the change of joint evaluation parameters, while elastic of fabric only affects momentum of elbow and wrist, but in general not obvious.

\subsection{Fabric Composite Measurement Experiment}

It is concluded above: compared to fabric elastic, the change in fabric thickness has a greater influence on parameters of each joint. In this experiment, the thickness of fabrics was changed by means of material composite ways to obtain the optimum protective material. Choosing optimal fabric $\mathrm{D}$ for experiment based on the elastic experiment. The thickness of fabric D was $0.6 \mathrm{~mm}\left(\mathrm{~A}_{1}\right)$, and meanwhile another sportswear fabrics with the thickness of $0.8 \mathrm{~mm}\left(\mathrm{~A}_{2}\right)$ and $1.0 \mathrm{~mm}\left(\mathrm{~A}_{3}\right)$ were selected as an experimental materials.

According to Fabrication of composite fabrics and garment technology (Zhou \& Wang, 2011), six composite ways of fabrics were set as follows (Fig. 8): double composite $\left(\mathrm{C}_{1}\right)$, double quilting compound $\left(\mathrm{C}_{2}\right)$, double-layer adhesive composite $\left(\mathrm{C}_{3}\right)$, double-layer quilting adhesive composite $\left(\mathrm{C}_{4}\right)$, double-layer with sponge compound $\left(\mathrm{C}_{5}\right)$ ( sponge thickness of $0.2 \mathrm{~mm}$ ), double layer quilting with sponge compound $\left(\mathrm{C}_{6}\right)$.

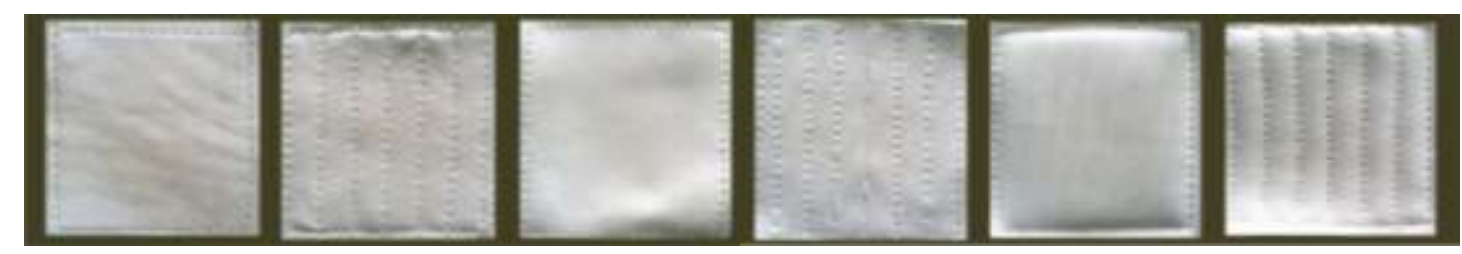

Figure 5. Six composite ways of clothing fabric : $\mathrm{C}_{1}, \mathrm{C}_{2}, \mathrm{C}_{3}, \mathrm{C}_{4}, \mathrm{C}_{5}, \mathrm{C}_{6}$, respectively.

According to six composite ways, the selected fabrics were made of shoulder pads, elbow, wrist, a total of 54 sets of protective equipment for tennis dynamic measurement experiments. Through inverse kinetic principle to calculate momentum and $\mathrm{MBC}$ of joints in $0.5 \mathrm{~s}$ after impact. The composites are independent variables, the momentum and MBC of each joint in the upper limb are strain variables, Value $\mathrm{P}$ is significance, discussing whether different composites of same fabric affect evaluation parameters in this paper. The results of the evaluation parameters are as follows: Tab. 5 is a single factor analysis of variance, where only value $\mathrm{P}$ is displayed. 
Table 5. Evaluation parameter changes with three thickness of fabrics in different composite methods

\begin{tabular}{llll}
\hline Evaluation parameter & Value $\mathrm{P}\left(\mathrm{A}_{1}\right)$ & Value $\mathrm{P}\left(\mathrm{A}_{2}\right)$ & Value $\mathrm{P}\left(\mathrm{A}_{3}\right)$ \\
\hline Shoulder-M & 0.028 & 0.003 & 0.045 \\
Elbow-M & 0.015 & 0.028 & 0.024 \\
Wrist-M & 0.043 & 0.033 & 0.058 \\
Shoulder-MBC & 0.042 & 0.012 & 0.039 \\
Elbow-MBC & 0.022 & 0.019 & 0.030 \\
Wrist-MBC & 0.014 & 0.010 & 0.001 \\
\hline
\end{tabular}

Six composite methods of $A_{1}(0.6 \mathrm{~mm})$ obviously affect the evaluation parameters of the three joints, especially on elbow. The most significant influence is $\mathrm{MBC}$ of the wrist. $\mathrm{A}_{2}(0.8 \mathrm{~mm})$ also has significant effects on the evaluation parameters, especially on value of shoulder momentum. Combined momentum and MBC, the influence of the shoulder is larger, but not on wrist. $\mathrm{A}_{3}(1 \mathrm{~mm})$ with different composites did not cause a significant change in wrist momentum, but its MBC was the most significant. Moreover, evaluation parameters of elbow change significantly. Based on the analysis of different composites, the composite patterns of the fabric have a significant effect on the change of the evaluation parameters. And the most distinguished influence is the evaluation parameters of the elbow.

\subsection{Protector Wrapping Experiment}

This experiment verified the protective performance of the fabric on human body, mainly through protector wrapping on elbow and wrist. On the basis of market research to design sports armor. Elbow selected cuff type $\left(\mathrm{E}_{1}\right)$ and bandage type $\left(\mathrm{E}_{2}\right)$, wrist selected cuff type $\left(\mathrm{W}_{1}\right)$ and winding type $\left(\mathrm{W}_{2}\right)$, and this experiment added forearm and big arm parts of the protective gear to improve the experimental data. The effect of different wrapping patterns on the change of parameters in each joint was compared by changing the style of gears.

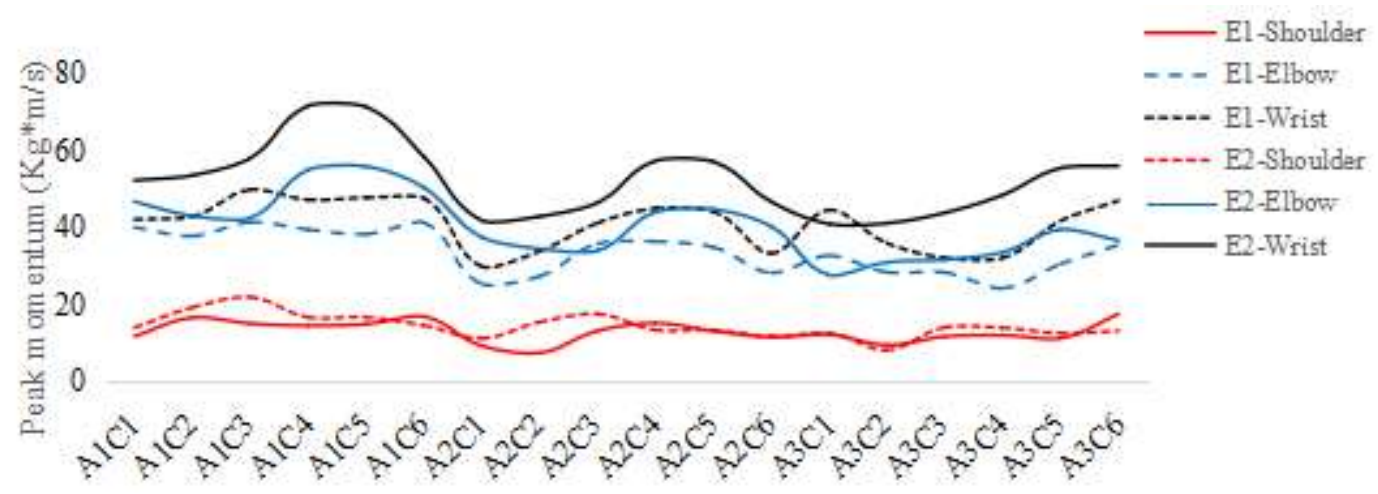

(a)

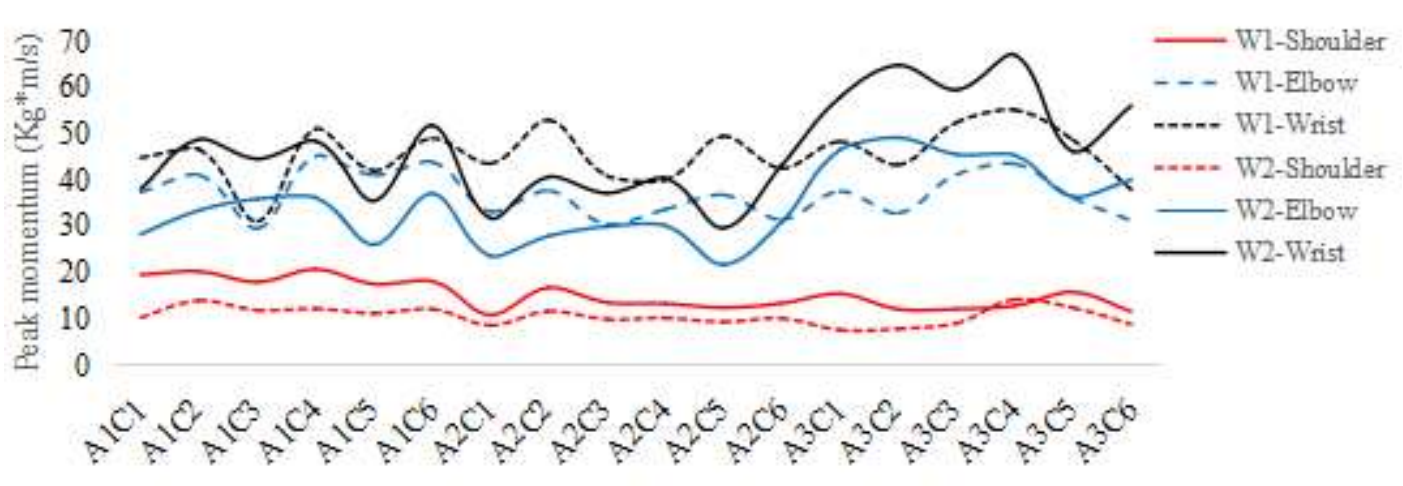

(b) 


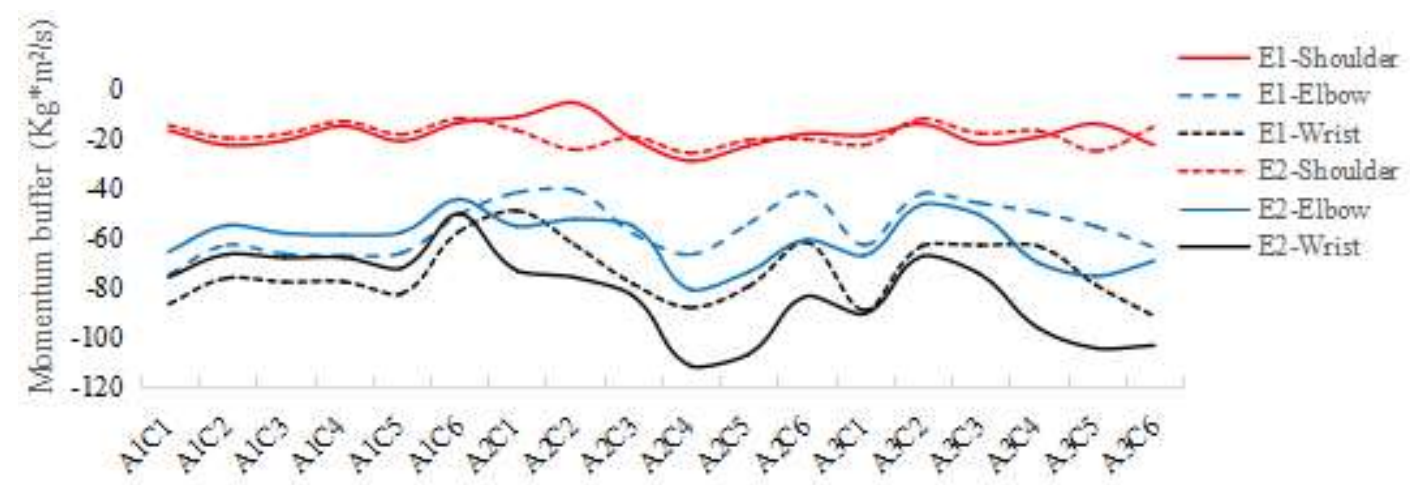

(c)

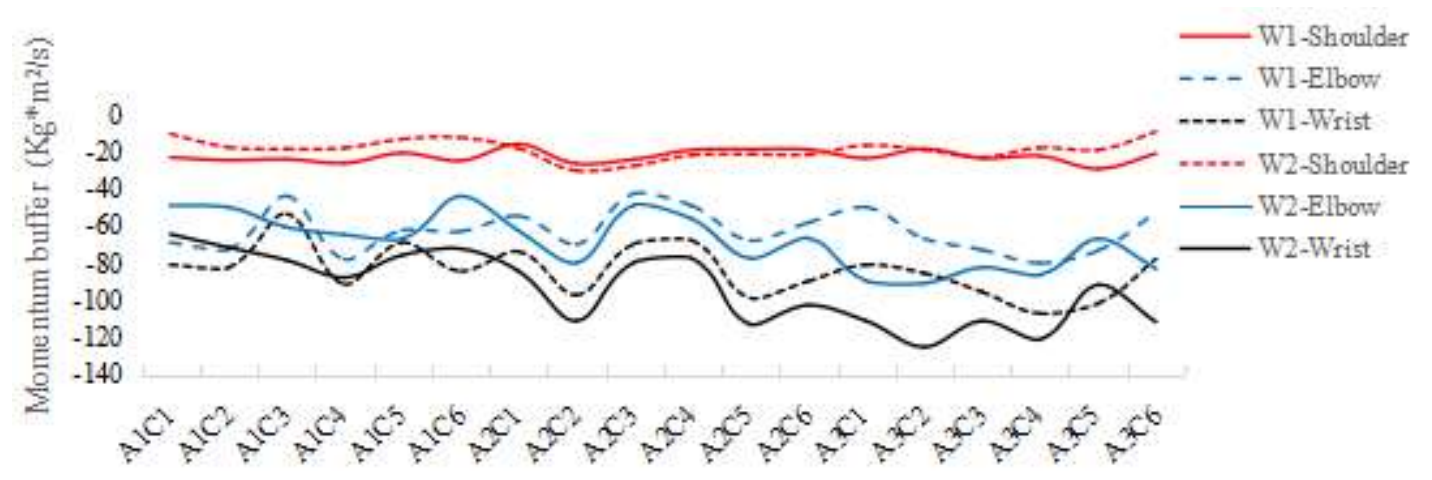

(d)

Figure 6. Peak momentum (a), (b) and MBC (c), (d)comparison of upper limb joints between $\mathrm{E}_{1}$ and $\mathrm{E}_{2}, \mathrm{~W}_{1}$ and $\mathrm{W}_{2}$.

The effect of two elbow pads have a consistent trend on peak momentum of each joint in different compound ways, besides shoulder in the sixth composite. The peak momentum of $E_{1}$ on each joint is less than that of $E_{2}$, Both type had large numerical fluctuation, $\mathrm{E}_{1}$ relatively functions well. While the $\mathrm{MBC}$ of $\mathrm{E}_{1}$ and $\mathrm{E}_{2}$ show a similar trend, but $\mathrm{E}_{1}$ is larger than $\mathrm{E}_{2}$ in wrist. As for wrist type, the data is uniform comparing peak momentum of shoulder and elbow, but the value of $\mathrm{W}_{1}$ was obviously smaller than $\mathrm{W}_{2}$ in wrist joint and shoulder joint. The MBC comparison $\mathrm{W}_{1}$ and $\mathrm{W}_{2}$ has a similar trend, it can't clarify which is better.

The above is a preliminary analysis of the pros and cons of the type of fixture, but it's hard to see the influence of pad types on the joint momentum and MBC. Tab. 6 is the analysis of significance on changing the pads wrapping type.

Table 6. Evaluation parameters changes with three thickness of fabrics in different wrapped types

\begin{tabular}{lccc}
\hline Evaluation parameter & Value $\mathrm{P}\left(\mathrm{A}_{1}\right)$ & Value $\mathrm{P}\left(\mathrm{A}_{2}\right)$ & Value $\mathrm{P}\left(\mathrm{A}_{3}\right)$ \\
\hline Shoulder-M & 0.000 & 0.000 & 0.000 \\
Elbow-M & 0.000 & 0.002 & 0.000 \\
Wrist-M & 0.000 & 0.000 & 0.000 \\
Shoulder-MBC & 0.000 & 0.000 & 0.000 \\
Elbow-MBC & 0.028 & 0.165 & 0.000 \\
Wrist-MBC & 0.527 & 0.058 & 0.000 \\
\hline
\end{tabular}

Wearing gears of different wrapping type, the peak momentum of each joint changes to a great extent, and the significance is obvious. The MBC of Elbow and wrist do not have significant changes when use $A_{1}$ and $A_{2}$. While peak momentum and $\mathrm{MBC}$ of $\mathrm{A}_{3}$ changes a lot. The main reason causing these results of the three fabrics is probably the thickness of the fabric.

In this section, four factors of fabric: elasticity, thickness, composite method and pads wrapping types were used as independent variables to analyze whether they had significant changes on evaluation parameters. The experimental data shows: these factors are of great significance to the change of the evaluation parameters of upper limb joints, while elbow evaluation parameters are the most obviously affected, and the wrapping ways of pads are important factors to peak momentum changes. 


\section{Conclusion}

By studying the principle of muscles protection on bones, this paper chose the spring oscillator model to present a simple physical protection model to show external auxiliary product and muscle's protection on bones. This paper used three dynamic experiments to verify the gears' protection on upper limb joints and its influence factors. Compared with elasticity, thickness of clothing materials is more significant to protection performance on upper limb joints. In addition, fabric composite methods and wrapping types of pads are important factors to peak momentum changes, especially for elbow. This research proves the clothing materials do have positive effect on protection performance, and they are of some significance to the research and design of protective sportswear.

This paper simplified muscle and external auxiliary products protection model, actually their inner relation is complicated. Future studies should consider tightness and shearing between muscles and sportswear, and whether the both can be treated as an integration. Other evaluation parameters related to human upper limb muscles, such as electromyography and vibration characteristics of soft tissues, can be further analyzed. At the same time, the emphasis should be laid on the quantitative relationship between clothing materials and protective parameters of the human body, to make it clear what are the conditions for achieving the optimum protective performance.

\section{References}

Chung, K. C., Lark, M. E. (2017). Upper Extremity Injuries in Tennis Players, Diagnosis, Treatment, and Management. Hand Clinics, 33, 175-186. https://doi.org/10.1016/j.hcl.2016.08.009

Editing Group for Sports Biomechanics. (2000). Sports Bio-mechanics (2rd ed.). Beijing: Higher Education Press.

Ellenbecker, T. S. (1991). A total arm strength isokinetic profile of highly skilled tennis players. Journal of Isokinetic Exercise Science, 1(9), 29.

Elliott, B., Fleisig, G., Nicholls, R., \& Escamilla, R. (2003). Technique effects on upper limb loading in the tennis serve. Journal of Science and Medicine in Sport, 6(1), 76-87. https://doi.org/10.1016/S1440-2440(03)80011-7

Escamilla, R., \& Andrews, J. (2009). Shoulder muscle recruitment patterns and related biomechanics during upper extremity sports. Sports Medicine, 39(7), 569-590. https://doi.org/10.2165/00007256-200939070-00004

Graham, B. (2006). New impact protection textile provides defense and comfort. China Textile Leader, 6(7), 29-31.

Johnson, C. D., McHugh, M. P., Wood, T., et al. (2006) Performance demands of professional male tennis players. $\mathrm{Br}$ J Sports Med, 40(8), 696-9 [discussion: 699]. https://doi.org/10.1136/bjsm.2005.021253

Kovacs, M. S. (2006). Applied physiology of tennis performance. $\operatorname{Br} J$ Sports Med, 40(5), 381-5. https://doi.org/10.1136/bjsm.2005.023309

Li, R. X. (2008). Social sports instructor training materials technology level one, two, three, pp. 207-211. Shenyang: Shenyang press.

Nordin, M., Frankel, V. H. (2001). Basic Biomechanics of the Musculoskeletal System (3rd ed.). New York, NY: LWW.

Okholm, K. K, Dor, F., Guillaume, M., et al. (2015). Medical reasons behind player departures from male and female professional tennis competitions. Am J Sports Med, 43(1), 34-40. https://doi.org/10.1177/0363546514552996

Palmer, R. M., Green, P. C. Energy absorbing material. U.S. Patent 7794827[P]. 2010-09-14.

Perry, J. (1983). Anatomy and biomechanics of shoulder in Throwing, Swimming, Gymnastics and Tennis. Journal of Clinical Sports Medicine, 2(2), 247-270.

Sanchis-Moysi, J., Dorado, C., Olmedillas, H., Serrano-Sanchez, J. A., \& Calbet, J. A. (2010). Bone and lean mass inter-arm asymmetries in young male tennis players depend on training frequency. European Journal of Applied Physiology, 110, 83-90. https://doi.org/10.1007/s00421-010-1470-2

Sciascia, A., Thigpen, C., Namdari, S., \& Baldwin, K. (2012). Kinetic chain abnormalities in the athletic shoulder. Sports Medicine \& Arthroscopy Review, 20(20), 16-21. https://doi:10.1097/JSA. 0b013e31823a021f

Wang, Z. Z., Ding, S., Wang, D. (2013). Design considerations on impact resistant sport protective products. Shandong Textile Science and Technology, 1, 43-46.

Wei, S. H., Chiang, J.Y., Shiang, T. Y., \& Chang, H. Y. (2006). Comparison of shock transmission and forearm electromyo-graphy between experienced and recreational tennis players during backhand strokes. Clinical Journal of Sport Medicine, 16(2), 129-135. https://doi.org/10.1097/00042752-200603000-00008

Zhou Luying, Wang Yueping. (2011). Modern Clothing Materials. Beijing: China Textile Press. 